NEWS

\section{Planet hunters lose}

\section{out to Hubble rescue}

A stronomers' elation at the prospect of a rescue for the Hubble Space Telescope is turning to dismay as the price of saving the venerable observatory becomes clear.

Last week, NASA turned in a revised budget plan to Congress that includes cuts and delays to several programmes, including the roving Mars Science Laboratory and searches for planets like Earth. The proposed cuts would also lead to belt-tightening in the Hubble project itself, where grants for guest observers would be reduced by an average of $13 \%$.

Two planet-hunting projects - the Space Interferometry Mission (SIM) and the Terrestrial Planet Finder (TPF) - have been deferred until as yet unspecified dates. SIM would orbit the Sun, measuring stars' positions and trying to detect planets similar to Earth. The TPF, which consists of two space-based observatories, would follow up SIM's findings between 2014 and 2020 with detailed spectral analyses.

Both projects have been scaled back before. SIM is currently undergoing another redesign in an attempt to keep its cost below $\$ 1.2$ billion. One possibility, says Charles Beichman of the Jet Propulsion Laboratory in Pasadena, California, who is on the SIM and TPF teams, is to advance its launch to 2010 , because shorter development times often reduce costs. But that now looks unlikely.

The TPF was among the top recommendations of a National Research Council panel that set priorities in $\mathbf{2 0 0 0}$ for the next decade of astronomy. But it is fraught with technical challenges - the panel called it " the most ambitious science mission ever attempted by NASA.

Deferring SIM and the TPF would be a blow to NASA's planetary search programme, which is already struggling with near-term projects. A plan to supplement the 10-metre ground-based optical Keck Telescopes, on Mauna Kea in Hawaii, with small 'outrigger' telescopes to detect Uranus-sized planets has been bogged down for years by battles with local culturalrights groups (see Nature 417, 5; 2002). And recent budget cuts have delayed the launch of the Kepler mission to look for very distant planets by eight months to June 2008.

Ground-based telescopes have found 155 planets around other stars and the European Space Agency lists the search for such 'extrasolar' planets among its priorities for the next decade. This leaves Beichman and others worried that NASA is neglecting a promising field.

Some are also anxious that the cost of a shuttle rescue mission to Hubble is squeezing

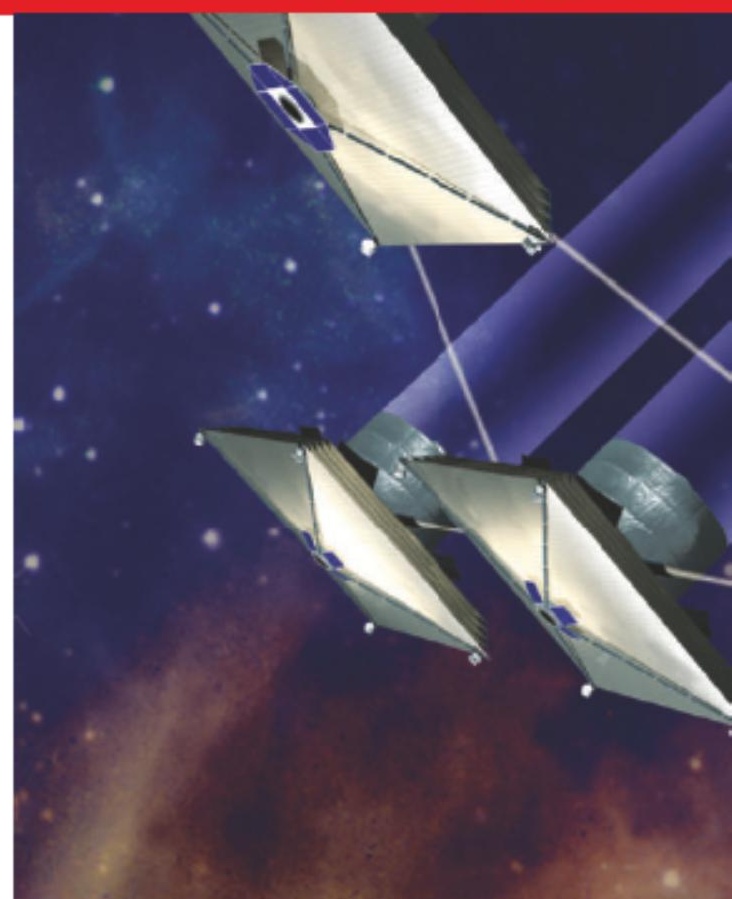

The Terrestrial Planet Finder will hunt for other Earths, if NASA ever finds the cash to get it off the ground.

- or giving NASA an excuse to squeeze other projects. Most astronomers would support saving Hubble if money were no object. But an increasing number agree with Nobel laureate and astrophysicist Joseph Taylor of Princeton University, New Jersey, who told a congressional committee: "I do not favour such a plan if it would require major delays or reordering of NASA's present science priorities."

David Black, chairman of the American Astronomical Society's public-policy committee, says the balance of opinion has shifted towards the idea that Hubble isn't worth the sacrifice of future missions. Yet he says the telescope is only one of NASA's financial

\title{
Large genomic differences explain our little quirks
}

COLD SPRING HARBOR, NEW YORK

When the finished sequence of the human genome was unveiled last year, biologists said that it told a story of harmony for the human family. Every one of us, it turns out, shares $99 \%$ of our DNA with all the other

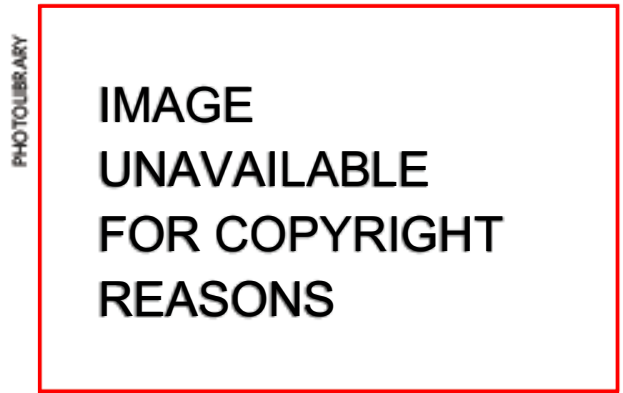

People'sgenomes just don't line up. people on Earth. But it's our differences that really fascinate us. And at last week's annual genome meeting in Cold Spring Harbor, New York, scientists revealed a wealth of data indicating a surprising conclusion about human diversity - much of it might be explained by large structural differences between individual genomes, not by tiny differences in individual genes.

Biologists used to think that our genomes all had the same basic structure - the same number of genes, in roughly the same order, with a few minor differences here and there in the sequence of DNA bases. Now, technologies comparing whole human genomes show that this picture is incomplete.

Two years ago, a group of researchers led by Michael Wigler at Cold Spring Harbor
Laboratory found the first evidence that some of us have more copies of certain genes than do others (R. Lucito et al. Genome Res. 13, 2291-2305; 2003). And at last week's meeting, Evan Eichler of the University of Washington in Seattle reported that this is just the beginning: not only do we carry different copy numbers of parts of our DNA, we also have varying numbers of deletions, insertions and other major rearrangements in our genomes.

In fact, Eichler found at least 297 places in the genome where different individuals have different forms of these major structural variations. At these spots, some of us might carry a major deletion, for example, or an extra hundred bases of DNA.

But do such differences mean anything? 